\title{
Prediction of helicopter rotor noise in hover
}

\author{
A.N. Kusyumov ${ }^{1, a}$, S.A. Mikhailov ${ }^{2}$, L.I. Garipova ${ }^{3}$, A.S. Batrakov ${ }^{4}$ and G. Barakos ${ }^{5}$ \\ ${ }^{1-4}$ Kazan National Research Technical University n.a. A. N. Tupolev (KNRTU-KAl), Kazan, Russia \\ ${ }^{5}$ University of Liverpool, Liverpool, UK
}

\begin{abstract}
Two mathematical models are used in this work to estimate the acoustics of a hovering main rotor. The first model is based on the Ffowcs Williams-Howkings equations using the formulation of Farassat. An analytical approach is followed for this model, to determine the thickness and load noise contributions of the rotor blade in hover. The second approach allows using URANS and RANS CFD solutions and based on numerical solution of the Ffowcs Williams-Howkings equations. The employed test cases correspond to a model rotor available at the KNRTUKAI aerodynamics laboratory. The laboratory is equipped with a system of acoustic measurements, and comparisons between predictions and measurements are to be attempted as part of this work.
\end{abstract}

\section{Introduction}

For a conventional helicopter, there are two fundamental elements that contribute to the generation of near-field and far-field noise, themain rotor and the tail rotor [1]. Engine and fuselage noise are typically of secondary significance. A helicopter main rotor generates primarily tonal and broadband noise. The tonal noise is usually divided into the deterministic components of thickness and loading noise, blade-vortex interaction (BVI) noise [2] and high-speed impulsive noise. Similarly, broadband noise consists of the non-deterministic loading noise sources classified as turbulence ingestion noise, bladewake interaction noise and blade self-noise [3].

BVI noise can be a dominant contributor and has a strong component below and ahead of the rotor. Basic loading noise during hover is generally dominant in a conical region directed 30 to 40 degrees [1] downward from the rotor plane, while broad-band noise radiates mostly out of the plane of the rotor.

Thickness noise and loading noise are described by linear aerodynamic theory. The reason of thickness noise is the displacementof the fluid by the rotor blade and loading noise is caused by the accelerating force generated by moving blade surface. The term "loading noise" is usually a reference to the harmonic noise from non-impulsive loading sources, whereas impulsive loading noise due to blade-vortex interaction.

In this work the focus is on the main rotor. The geometry of the rotor employed for theoretical analysis corresponds to the helicopter rig rotor of KNRTU-KAI aerodynamics laboratory. Acoustic and aerodynamic tests to study this rotor are anticipated in the near future. URANS and RANS CFD solutions will be used also as initial data for numerical solution of the Ffowcs Williams-Howkings equations. The main goal of this work is a preliminary research of the acoustics of the KNRTU-KAI rotor rig.

\section{Mathematical formulation of task}

A widely used method for predicting the aerodynamically generated rotor noise is based on the Ffowcs Williams Hawkings $(\mathrm{FW}-\mathrm{H})$ equation [4]. Implementation of the FW-H equation to CFD methods is usually basedon the integral formulation of the $\mathrm{FW}-\mathrm{H}$ equation. The far field retarded-time formulation by Farassat is commonly referred to as Formulation 1 [5]:

$$
4 \pi p^{\prime}(\mathbf{x}, t)=\frac{1}{a_{0}} \frac{\partial}{\partial t} \int_{f=0}\left[\frac{\rho_{0} a_{0} v_{n}+l_{r}}{r\left|1-M_{r}\right|}\right]_{r e t} d S
$$

Here $\mathrm{a}_{0}$ is sound speed; $f=0$ is a function that describes the source surface; $\rho_{0}$ is the density of medium; $v_{n}$ is the local normal velocity of source surface; $M_{r}=M_{i} \hat{r}_{i}$ is Mach number of source in radiation direction; $r$ is the distance between observer and source, $r=|\mathbf{x}-\mathbf{y}|, \mathbf{x}$ is the observer position vector, with components $x_{i}, \mathbf{y}$ is the source position vector, with components $y_{i} ; p^{\prime}=p-p_{0}$ is the acoustic pressure, $p_{0}$ is the pressure of the medium, $p$

\footnotetext{
${ }^{1 \mathrm{a}}$ Prof., department of aero-hydrodynamics, postbox7@mail.ru

${ }^{2}$ Prof., department of aero-hydrodynamics, michailov@kai.ru

${ }^{3} \mathrm{PhD}$ student, lyaysan_garipova@mail.ru

${ }^{4} \mathrm{PhD}$ student, batrakov a.c@mail.ru

${ }^{5}$ Prof., department of Engineering, G.Barakos@liverpool.ac.uk
} 
is the surface pressure; $l_{i}$ are the components of the local force intensity, $l_{r}=l_{i} \hat{r}_{i}$. The subscript ret denotes the retarded time and the integration is performed over the actual blade surface $f=0$. For a moving surface the retarded time $\tau$ is determined by the expression

$$
|\mathbf{x}-\mathbf{y}(\eta, \tau)|=a_{0}(t-\tau),
$$

where $t$ is the source or emission time, and $\eta$ is the Lagrangian variable of a point on the moving surface (figure 1, [6]).

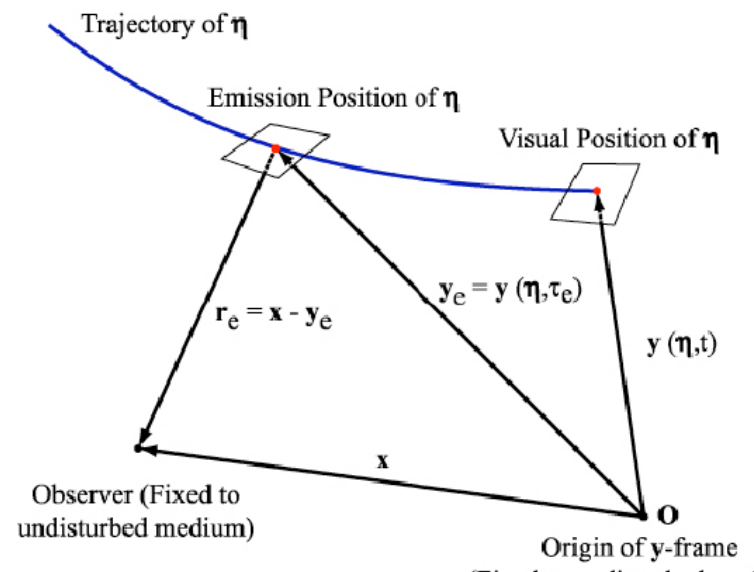

(Fixed to undisturbed medium)

Figure 1. Schematic of the trajectory of a source point $\eta$ relative to an observer fixed inside the medium ( $\mathbf{x}$ - or $\mathbf{y}$-frame) and definitions of visual and emission positions of the source [6].

Equation (1) can be rewrite in the form $[5,6]$ :

Here

$$
p^{\prime}(\mathbf{x}, t)=p^{\prime}(\mathbf{x} ; t)+p_{L}^{\prime}(\mathbf{x} ; t) .
$$

$$
p_{T}^{\prime}(\mathrm{x} ; t)=\frac{1}{4 \pi} \frac{\partial}{\partial t} \int_{f=0}\left[\frac{\rho_{0} v_{n}}{r\left|1-M_{r}\right|}\right]_{r e t} d S
$$

is the thickness noise, and

$$
p^{\prime}{ }_{L}(\mathbf{x} ; t)=\frac{1}{4 \pi a_{0}} \frac{\partial}{\partial t} \int_{f=0}\left[\frac{l_{r}}{r\left|1-M_{r}\right|}\right]_{r e t} d S
$$

is the load noise. Equation (3) can also be rewritten in the form [6]:

$$
p_{L}^{\prime}(\mathbf{x} ; t)=\frac{1}{4 \pi a_{0}} \frac{\partial}{\partial t} \int_{f=0}\left[\frac{p \cos \theta}{r\left|1-M_{r}\right|}\right]_{r e t} d S
$$

where $\cos \theta=n_{i} \hat{r}_{i}$, i.e., $\theta$ is the local angle between the normal to the surface and the radiation direction $\hat{r}_{i}$ at the emission time.

For a hovering rotor of radius $R$ the singularity point, emitted a disturbance at position "P" with local blade radius $r_{P}\left(r_{P}<R\right)$, travels at the ambient speed of sound and arrives at the observer's position " 0 " with the time delay $r / a_{0}$. The observer time is related to emission time by:

$$
\tau=t-r(t, \tau) / a_{0}
$$

or

$$
\Omega \tau=\psi=\Omega t-\Omega r / a_{0}
$$

where $\Omega$ is the angular velocity of rotor.

For an observer located at the distance $r_{H}$ to the rotor plane the expression for the distance "observer-source point" has the form:

$$
r^{2}=\left(r_{H}+r_{P} \cos \psi\right)^{2}+\left(r_{P} \sin \psi\right)^{2}
$$

Transfer to the retarded time $\tau$ in the integrals (3), (5) leads to:

$$
d \tau / d t=1 /\left(1-M_{r}\right)
$$

where $M_{r}=\Omega d r / d t$ and the term $1 /\left(1-M_{r}\right)$ is the Doppler factor.

\section{Analytical solutions for observer located at the rotor disk plane}

The analytical solution is based on method of the reference [8] and the expression (3) for thickness noise can be written in the form:

$$
p_{T}^{\prime}(\mathbf{x}, t)=\frac{\rho_{0} a_{0}^{2}}{2} F_{H} F_{\varepsilon} T_{M} .
$$

Here $F_{H}=R / r_{H}$ is the distance factor, $F_{\varepsilon}=A \varepsilon / A$ is the aerofoil shape factor and $T_{M}$ is the thickness factor:

$$
\begin{gathered}
T_{M}\left(\psi, M_{H}\right)=\frac{M_{H}^{3}}{12} \times \\
\times\left\{\begin{array}{c}
-\frac{\left(3-M_{H} \sin \psi\right) \sin \psi}{\left(1-M_{H} \sin \psi\right)^{3}}+\frac{M_{H} \cos ^{2} \psi}{10\left(1-M_{H} \sin \psi\right)^{4}} \times \\
\times\left[\begin{array}{c}
50+39 M_{H}^{2}-45 M_{H} \sin \psi-11 M_{H}^{2} \sin ^{2} \psi+ \\
12 M_{H}^{3} \sin \psi-18 M_{H}^{3} \sin ^{3} \psi
\end{array}\right]
\end{array}\right\}
\end{gathered}
$$

In expression (8) $\rho_{0}$ is the air density, $a_{0}$ is the sound speed, $M_{H}$ is the tip Mach number, $\psi$ is the local azimuth angle, $A$ is the rotor disk area, $A \varepsilon$ is the aerofoil cross sectional area.

For a rotor with $N_{b}$ blades and thrust $T$, the expression (5) for the load noise can be written as:

$$
p^{\prime}{ }_{L}(\mathbf{x} ; t)=\frac{\rho_{0} a_{0}^{2}}{2} F_{H} F_{T} L_{M} .
$$

Here $F_{T}$ is the trust factor, corresponded to the expression:

$$
F_{T}=\frac{1}{60 \sqrt{2} N_{b}}\left(\frac{T}{\rho_{0} a_{0}^{2} A}\right)^{3 / 2},
$$

and $L_{M}$ is the load factor:

$$
\begin{gathered}
L_{M}\left(\psi, M_{H}\right)=\cos \psi\left(1-M_{H} \sin \psi\right)^{-3} \times \\
\left(\begin{array}{c}
60+30 M_{H}^{2} \cos ^{2} \psi-120 M_{H} \sin \psi- \\
30 M_{H}^{3} \sin ^{2} \cos ^{2} \psi+80 M_{H}^{2} \sin ^{2} \psi+ \\
9 M_{H}^{4} \sin ^{2} \psi \cos ^{2} \psi-20 M_{H}^{3} \sin ^{3} \psi
\end{array}\right)
\end{gathered}
$$

General form of azimuthal distribution of the rotor total noise is determined by the $T_{M}$ and $L_{M}$ functions, presented in the figures 2,3 . 


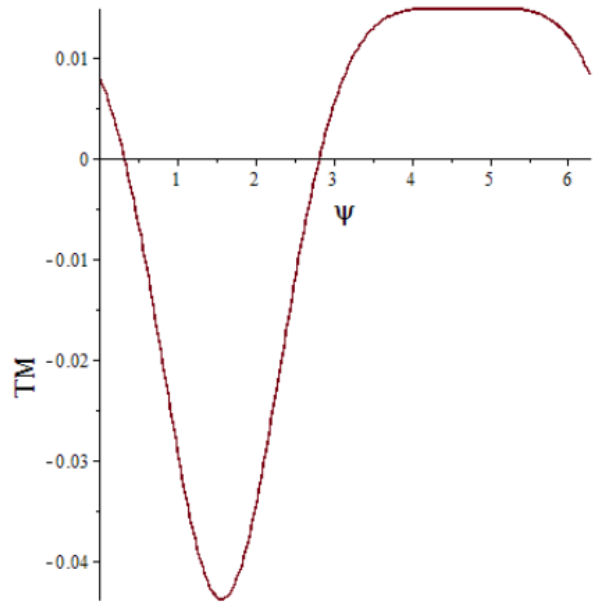

a)

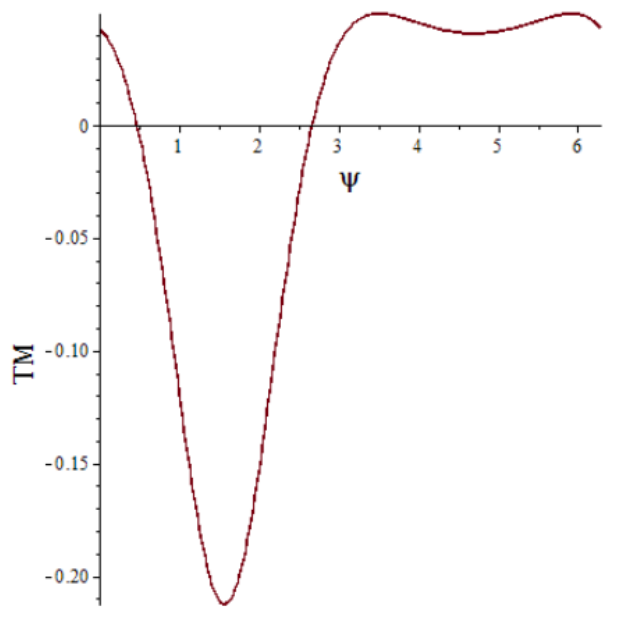

b)

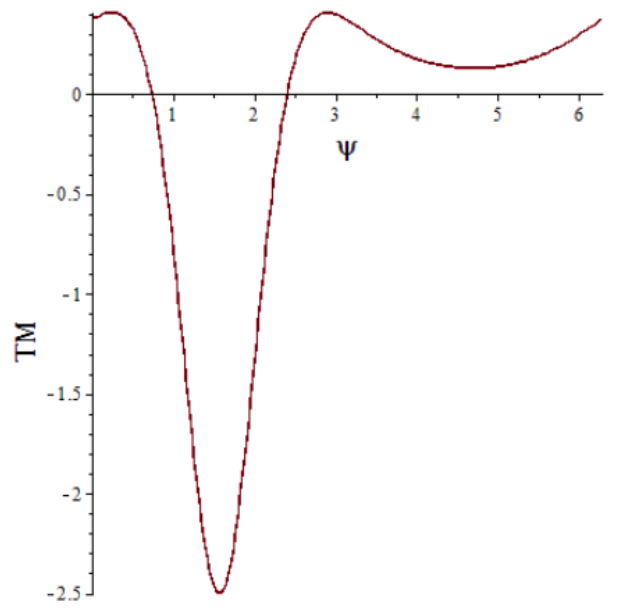

c)

Figure 2. Azimuthal distribution (source time) of the shape factor $T_{M}$ for different values of $M_{H}$ : a) $M_{H}=0.2$; b) $M_{H}=0.3$; c) $M_{H}=0.5$.

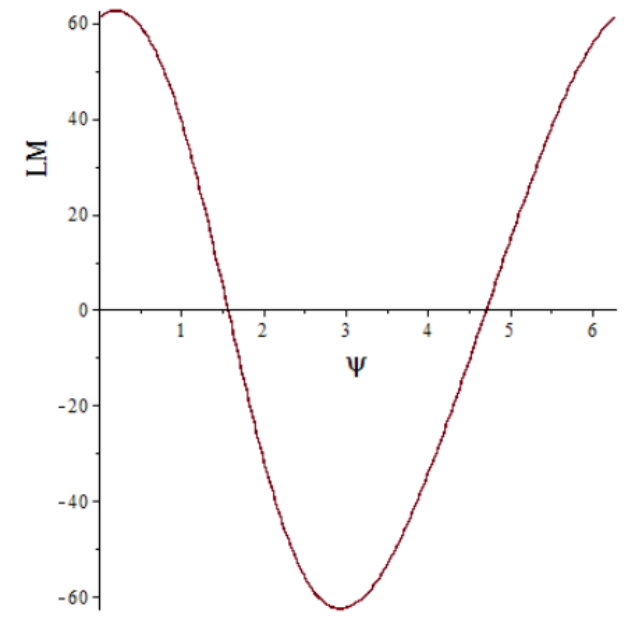

a)

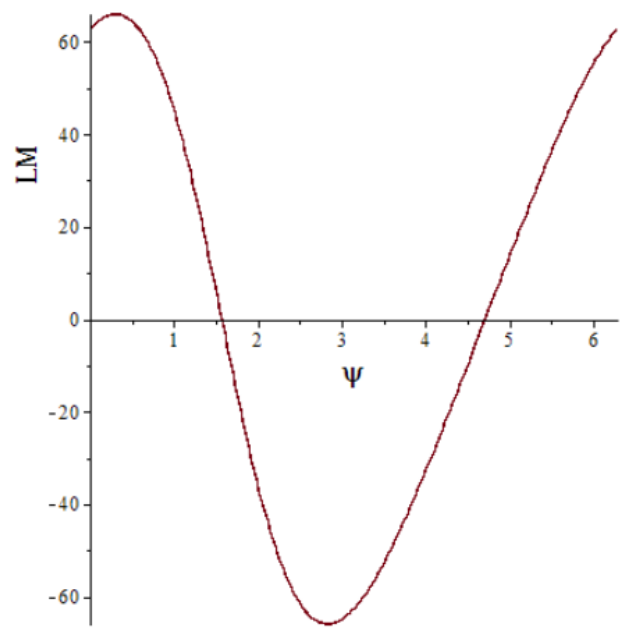

b)

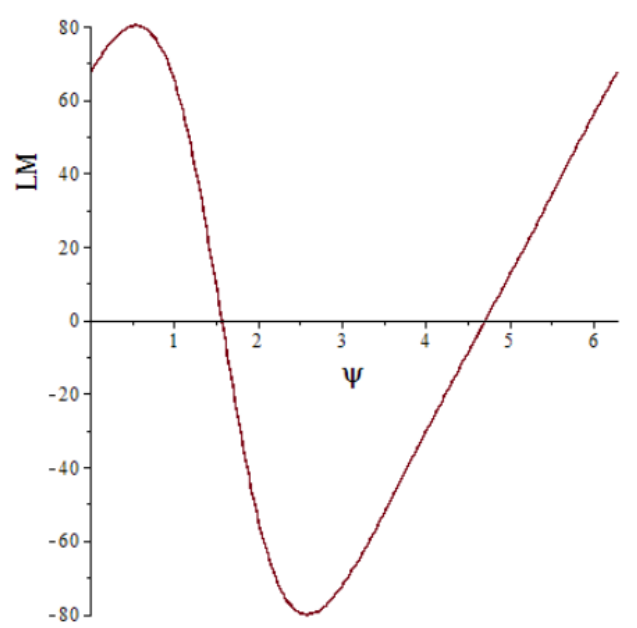

c)

Figure 3. Azimuthal distribution (source time) of the load factor $L_{M}$ for different values of $M_{H}$ : a) $M_{H}=0.2$; b) $M_{H}=0.3$; c) $M_{H}=0.5$. 
Using functions $T_{M}\left(M_{H}, \psi\right)$ and $L_{M}\left(M_{H}, \psi\right)$ and expressions (8), (9) it is possible to determine the hover rotor sound pressure level for an observer, located at the rotor disk plane. Figure 4 presents simulation results for the sound pressure level (for observer time) under the conditions: NACA 0012 aerofoil; rotor radius $R=8 \mathrm{~m}$; hover tip Mach number $M_{H}=0.82$; rotor thrust $T / A=6 \mathrm{psf}(287.28 \mathrm{~Pa})$; observer distance from rotor hub $r_{H}=800 \mathrm{~m}$. Simulations results obtained using formulas (2), (8), (9) are compared to the data presented inreference [8]. Comparison of the results shows that the results of total noise modeling correspond to the data of reference [8], thus the load noise component (it was admitted the constant rotor disk surface load distribution) is slightly under predicted.

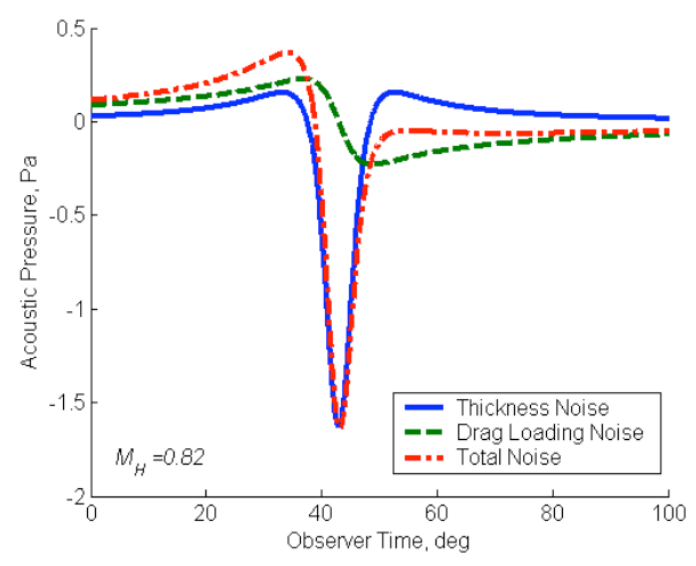

a)

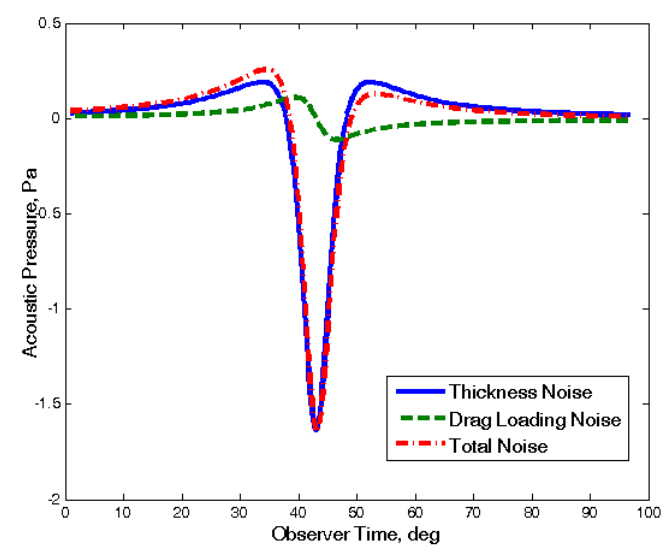

b)

Figure 4. Rotor blade noise sound pressure level for hover mode: a) reference [8]; b) according to formulas (2), (8), (9).

\section{Validation of program for solution of FWH equations}

Numerical simulation of FWH equations was realized using a FORTRAN computer code. Validation of the computer code for FWH equations was conducted using an analytical solution for the conditions of reference [9]. Reference [9] presents data of experimental study of acoustic radiation of two blades rotor with parameters of study: NACA 0012 aerofoil; rotor radius $R=2.1 \mathrm{~m}$; hover tip Mach number $M_{H}=0.8$; rotor thrust $T / A=6 \mathrm{psf}$ $(287.28 \mathrm{~Pa})$; observer distance from rotor hub $r_{H}=6.25 \mathrm{~m}$. Figure 5 presents the comparison of experimental data with the data predicted using analytical and numerical approaches in comparison to experimental data and data of reference [9]. The load noise component was determined for constant load distribution on the rotor disk surface. Figure 5 shows a 2 milliseconds time interval for the rotor period of 24 milliseconds.

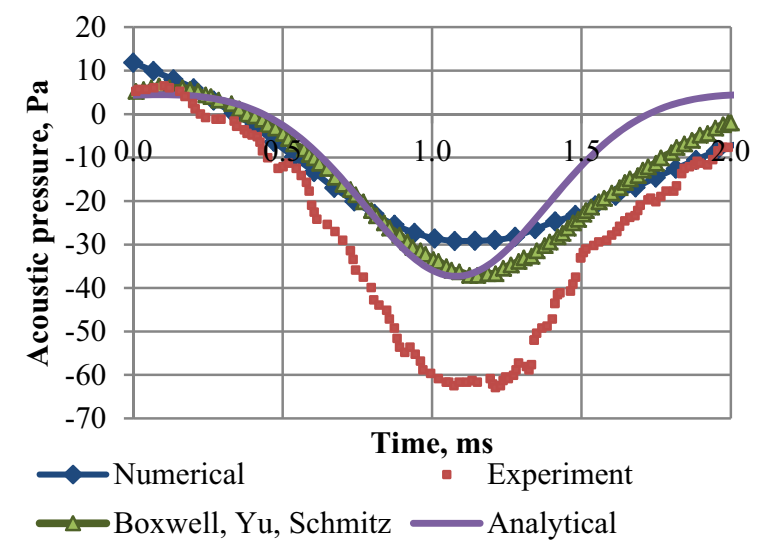

a)

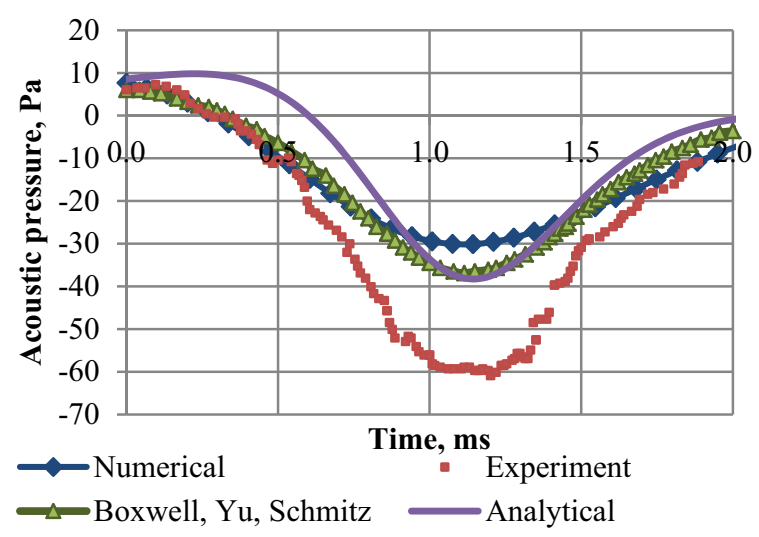

b)

Figure 5. Comparison of experiment and theory for the thickness and total rotor blade noise sound pressure level: a) thickness noise; b) total noise.

The difference between analytical and numerical solutions is determined by the different character of accepted admissions. There is a difference at the peak negative pressure levels between the linear monopole theory and experiment. The theoretical model underestimates the peak of negative-pressure level. Similar conclusions were noted in reference [10] for a high-speed rotor in forward flight from order-ofmagnitude and pulse-shape arguments. The major conclusion is that the linear acoustic analyses do not adequately describe the in-plane noise radiation processes from a high-tip-speed hovering rotor. 


\section{Numerical simulation of the helicopter rig using the $\mathrm{FWH}$ equations. Conclusion}

The helicopter rig (figure 6) of the KNRTU-KAI has a four bladed "ROTOR-G" rotor.

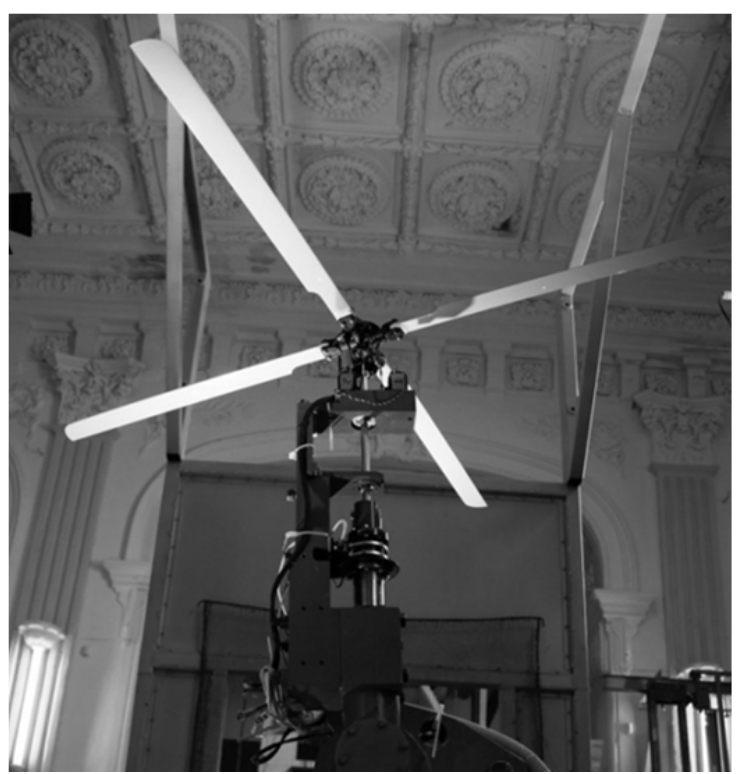

Figure 6. Experimental rig with the "ROTOR-G" rotor in experimental hall.

Some geometrical parameters of the rotor are given in Table 1. A CAD model of the "ROTOR-G" surface was obtained using a 3D laser scanner.

Table 1. Rotor geometry.

\begin{tabular}{|c|c|}
\hline \multicolumn{2}{|c|}{ Rotor Geometry } \\
\hline Number of blades, $N$ & 4 \\
\hline Diameter of rotor, $D=2 R(\mathrm{~m})$ & 1.5 \\
\hline Twist of blade & No \\
\hline Chord of main part of the blade, $c(\mathrm{~mm})$ & 52.8 \\
\hline $\begin{array}{c}\text { Thickness of the blade aerofoil, in } \% \text { of } \\
\text { the chord }\end{array}$ & 12 \\
\hline
\end{tabular}

Figure 7 presents the preliminary results of the rig rotor blade noise simulation. The numerical simulation results underestimate the peak of negative-pressure level in comparison with the analytical solution. Nevertheless both approaches demonstrated approximately similar expected noise level values for the range of azimuth angle. Computations were performed for the conditions: rotor radius $R=0.75 \mathrm{~m}$; hover tip Mach number $M_{H}=$ 0.8 ; rotor thrust $T / A=287.28 \mathrm{~Pa}$; observer distance from rotor hub $r_{H}=2.5 \mathrm{~m}$.

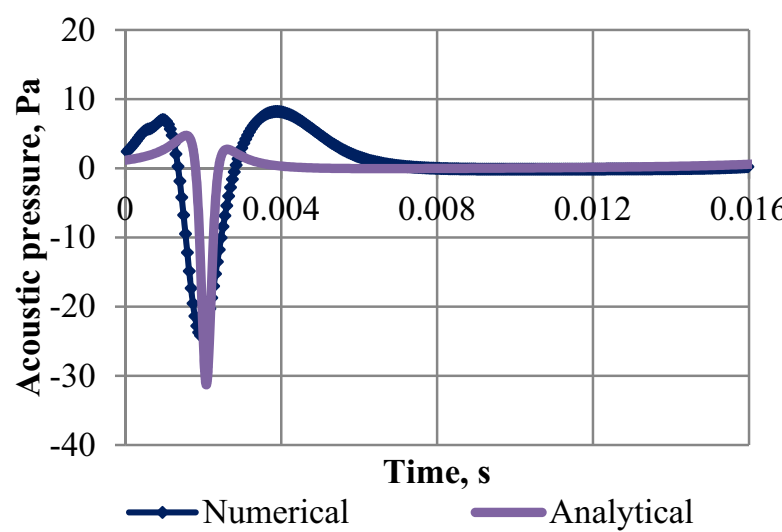

Figure 7. Comparison of numerical and analytical solution for the total rotor blade noise.

In the future both approaches (numerical and analytical) will be revised to correct some admissions of mathematical formulation of task and to extend areas of application of algorithms for out rotor plane location of observer.

This work is supported by the "Project part of state task in the field of the scientific activity" Grant (No 9.1694.2014/K).

\section{References}

1. B. Edwards, Ch. Cox, NASA/CR-2002-211650 (May 2002).

2. A. F. Deming, NACA TM 679 (1938).

3. K. S. Brentner, Modeling aerodynamically generated sound of helicopter rotors. Preprint submitted to Progress in Aerospace Sciences. $89 \mathrm{Pp}$.

4. J. E. Ffowcs Williams, D. L. Hawkings, Philosophical Transactions of the Royal Society, A 264 (1151), (1969).

5. K. S. Brentner, F. Farassat, Progress in Aerospace Sciences, 39. (2003).

6. F. Farassat, NASA/TM-2007-214853.

7. F.H. Shmitz and Y.H., Yu, NASA Technical memorandum 84390.

8. G. Gopalan, F.H. Shmitz, AHS Specialist's Conference on Aeromechanics, San Francisco, CA (Jan. 23-25, 2008).

9. D. A. Boxwell,Y. H. Yuand F. H. Schmitz, NASA CP-2052, (1978) and Vertica, 3, no. 1 (1979).

10. F. H. Schmitz and Y. H. Yu, J. Am. Helicopter SOC., 24, no. 1 (1979). 